\title{
Comparative mapping of chalkiness components in rice using five populations across two environments
}

\author{
Bo Peng ${ }^{1}$, Lingqiang Wang ${ }^{1 *}$, Chuchuan Fan¹, Gonghao Jiang ${ }^{1}$, Lijun Luo ${ }^{2}$, Yibo Li ${ }^{1}$ and Yuqing He $\mathrm{H}^{1 *}$
}

\begin{abstract}
Background: Chalkiness is a major constraint in rice production because it is one of the key factors determining grain quality (appearance, processing, milling, storing, eating, and cooking quality) and price. Its reduction is a major goal, and the primary purpose of this study was to dissect the genetic basis of grain chalkiness. Using five populations across two environments, we also sought to determine how many quantitative trait loci (QTL) can be consistently detected. We obtained an integrated genetic map using the data from five mapping populations and further confirmed the reliability of the identified QTL.

Results: A total of 79 QTL associated with six chalkiness traits (chalkiness rate, white core rate, white belly rate, chalkiness area, white core area, and white belly area) were mapped on 12 chromosomes using five populations (two doubled haploid lines and three recombinant inbred lines) across two environments (Hainan in 2004 and Wuhan in 2004). The final integrated map included 430 markers; 58.3\% of the QTL clustered together (QTL clusters), 71.4\% of the QTL clusters were identified in two or more populations, and $36.1 \%$ of the QTL were consistently detected in the two environments. The QTL could be detected again and showed dominance ( $q$ WBR1, qWBR8, qWBR12, and $q C R 5)$ or overdominance effects ( $q W C R 7)$ for the rate of the white belly or white core, respectively, and all four QTL clusters derived from Zhenshan 97 controlling white belly rate were stably and reliably identified in an $F_{2}$ population.

Conclusions: Our results identified 79 QTL associated with six chalkiness traits using five populations across two environments and yielded an integrated genetic map, indicating most of the QTL clustered together and could be detected in different backgrounds. The identified QTL were stable and reliable in the $F_{2}$ population, and they may facilitate our understanding of the QTL related to chalkiness traits in different populations and various environments, the relationships among the various chalkiness QTL, and the genetic basis for chalkiness. Thus, our results may be immediately used for map-based cloning of important QTL and in marker-assisted breeding to improve grain quality in rice breeding.
\end{abstract}

Keywords: Oryza sativa L., QTL, Rice, Chalkiness, Comparative mapping

\section{Background}

Rice (Oryza sativa L.) is one of the most important food crops worldwide and contributes to $40 \%$ of the total calorie intake for Chinese people. With the improvement of living standards, there is an increasing demand for better grain quality [1-3]. Rice grain quality is a complex

\footnotetext{
* Correspondence: lqwang@mail.hzau.edu.cn; yqhe@mail.hzau.edu.cn ${ }^{1}$ National Key Laboratory of Crop Genetic Improvement, National Center of Plant Gene Research and National Center of Crop Molecular Breeding, Huazhong Agricultural University, Wuhan 430070, China

Full list of author information is available at the end of the article
}

character with several components, including grain appearance and milling, eating, cooking, and nutritional qualities; appearance quality is mostly determined by grain shape and endosperm opacity (or chalkiness) [3,4]. Chalkiness is divided into white belly, white core (WC), and white back (WB) in rice grain, depending upon its location on or within the endosperm. It represents a major problem in rice production in many rice-producing areas of the world because chalkiness results in inferior milling, cooking, eating, and nutritional quality [1,5-11]. Thus, grain chalkiness 
determines grain quality and price, and its reduction is an important goal of artificial breeding in rice.

Previous studies have shown that chalkiness is a complex quantitative trait that is controlled by polygenes and readily influenced by environmental factors [12-14]. During the last 15 years, many molecular marker-based QTL analyses of rice grain chalkiness have been conducted [10,11,15-23], and 82 QTL have been detected using numerous mapping populations (http://archive.gramene.org/qtl/). Therefore, great progress has already been made toward understanding the genetic basis of chalkiness; however, no gene controlling the trait has been cloned, and not much is known about the genetic mechanisms for crop genetic improvement.

Almost every previous experimental population for studying chalkiness was limited in size, restricted to a cross, or resulted in low-density maps, and most previous samples were planted in specific environments [15-19,24-29]. Two problems are apparent from previous studies. First, the QTL effects detected in a single population are extremely limited for detection of QTL with desirable power; accuracy depends on the genetic diversity in the parental lines, heritability of the traits, the size of the crosses, as well as the density of genetic markers [30]. Second, identifying the alignments and allelism of multiple QTL across populations is difficult because each study was based on different experimental populations, markers, data collection, and analytical methods, although comparative analysis has been confirmed as an effective way to identify multiple alleles and to collect a great quantity of research information from different studies $[31,32]$. In addition, little attention has been paid to the components of chalkiness, which have different genetic bases.

In this study, four populations derived from four crosses between a common female parent Zhenshan 97 (ZS97) and four male parents (H94, Delong 208 [DL208], Nanyangzhan [NYZ], and Wuyujing [WYJ]) with different degrees of chalkiness and diverse genetic backgrounds were used to detect QTL for chalkiness traits across two environments by using a series of simple sequence repeat (SSR) markers. Comparative mapping analysis was conducted by making use of another genetic population derived from ZS97 and Minghui 63 (MH63) in our laboratory [16,33]. Our experiment involved a systematic analysis of the genetic basis of grain chalkiness in rice. Additionally, we endeavored to confirm the genetic basis of the internal components of chalkiness to determine whether they have different behaviors in various genetic backgrounds and environments. Furthermore, to verify the reliability of QTL, we developed an $\mathrm{F}_{2}$ population derived from a cross between ZS97 (high-chalkiness rate) and WG97 (low-chalkiness rate). Thus, a systematic analysis of the genetic basis of grain chalkiness has paved the way for molecular marker-assisted selection and map-based cloning of important genes and QTL, as well as the genetic improvement of grain chalkiness and grain quality in rice and potentially other staple crops.

\section{Methods}

\section{The mapping populations and the field experiment}

The five mapping populations included two doubled haploid (DH) lines (ZS97/H94 and ZS97/WYJ) and three recombinant inbred lines (RILs; ZS97/DL208, ZS97/NYZ, and ZS97/MH63) that were derived from three crosses; ZS97 (Oryza sativa L. ssp. indica) was their common female parent [16,34-38]. An $F_{2}$ population was derived from a cross between ZS97 and WG97 [39]. A total of six populations were used in this study.

The DH populations were derived from the cross of ZS97 with H94 (an indica variety with translucent endosperm) and WYJ (a japonica variety with a similar degree of chalkiness as ZS97) [35,38]. RIL population ZS97/DL208 was derived from a cross between ZS97 and DL208 (dull endosperm). ZS97/NYZ was derived from a cross between ZS97 and NYZ (a kind of indica red rice with greater chalkiness than ZS97), and the population ZS97/MH63 was derived from a cross between ZS97 and MH63 [16]. All populations and their parents were planted during the rice-growing season on the experimental farm of Huazhong Agricultural University in 2004 in Hainan and Wuhan, respectively. The day length in Hainan is longer than that in Wuhan (about 13 hours), while the temperature is much hotter in Wuhan $\left(36-39^{\circ} \mathrm{C}\right)$ than that in Hainan $\left(25-28^{\circ} \mathrm{C}\right)$.

An $F_{2}$ population (396 individuals, then knocked-out a major QTL from 1398 individuals) was derived from a cross between ZS97 and WG97 (backcross female parent without chalkiness or with low-rate chalkiness, O. sativa L. ssp. indica) [39]. This $\mathrm{F}_{2}$ population and the parents were planted during the rice-growing season in 2008 on the experimental farm of Huazhong Agricultural University, Wuhan, China.

Each line was planted with two replications in each sowing, with each line containing 10 plants transplanted in a single row with $16.5-\mathrm{cm}$ plant spacing and $26.4-\mathrm{cm}$ row spacing. Field management essentially followed normal agricultural practice [35]. Field irrigation was maintained to avoid drought stress to the late-maturing lines.

\section{Traits}

One hundred milled rice grains including broken grains were randomly selected from the middle six plants for each line and were put on a visualizer to identify those complying with the National Standard of the People's Republic of China-Good Quality of Rice Grains (GB/ T17891-1999). The grains with chalkiness were counted, 
and the percentage of chalky grains was calculated as the chalkiness rate (CR). For chalkiness area (CA), 20 grains with chalkiness were randomly selected, and the ratio of the CA to the whole kernel square for each grain was evaluated by visual assessment. Grains with WC and WB were further separated and counted. The parameters of white belly rate (WBR), white belly area (WBA), white core rate (WCR), and white core area (WCA) of WB and WC were estimated with the same method used for $\mathrm{CR}$ and CA. WCA and WBA could scarcely be distinguished from one another in ZS97 and NYZ because the areas were large and often fused together. All parameters for each sample of the lines and their parents were measured with two replications.

\section{DNA markers and assays}

Polymorphic SSR markers involving all 12 chromosomes were detected for genotypes in the four populations: 218 for $\mathrm{DH}$ population ZS97/H94, 179 for RIL population ZS97/DL208, 190 for RIL population ZS97/NYZ, and 179 for RIL population ZS97/WYJ [36]. The primers of the RM series were designed based on previous studies $[40,41]$, and those of the MRG series relied on the rice genome sequences of Monsanto Company [42]. The SSR assay was conducted as described previously [36].

\section{Data analysis}

All genetic linkage maps were constructed by Mapmaker 3.0 [43]. The average of the measurements for each line in each population was utilized for QTL analysis. QTLMapper version 1.6 was based on a mixed linear model approach $[44,45]$, and it was also employed to detect QTL containing the chalkiness traits of location-related chalkiness. In this analysis, likelihood ratio (LR) value $P=0.005$ (equivalent to $\mathrm{LOD}=4.03$ for $\mathrm{df}=6$ ) was utilized as the threshold for claiming the presence of putative main QTL. The significance of the QTL effects was further tested by Bayesian analysis $(P<0.005)$. The peak points of the LR in the linkage map were taken as the putative positions of the effects. Dominant and overdominant effect analysis in the ZS97/WG97 population was taken as described previously [46]. The relative contribution of a genetic component was calculated as the proportion of phenotypic variance explained in the selected model.

\section{Integrated genetic map}

An integrated genetic map was obtained using the JoinMap $^{\oplus} 4.0$ software program [47]. Information from the five mapping populations was integrated by the recombination frequencies and LOD values. Fixed order option was used to define the ordering of doubtful markers. The final integrated map included 430 markers.

\section{Results}

Measurements and segregations of the six chalkiness traits in four populations

Chalkiness traits of the parents (ZS97, H94, NYZ, and WYJ) and populations (ZS97/H94, ZS97/DL208, ZS97/ NYZ, and ZS97/WYJ) in Hainan and Wuhan are shown in Table 1. There were no observations for the chalkiness traits in parent DL208 because all endosperms were opaque. The chalkiness traits in parent NYZ were observed in $100 \%$ of the samples and were much more prominent than those in other parents for CR, WCR, and WBR. The WCA and WBA were large and often overlapped; therefore, it was difficult to estimate size. There was a smaller amount of chalkiness area in H94, and the endosperms were relatively transparent; ZS97 and WYJ had a larger amount of chalkiness area, and grain WC was readily distinguishable from grain WB. In addition, the tremendous transgressive segregation phenomenon of traits was also observed in the four populations (Table 1). After the grain WC analysis of WB and other chalkinessrelated traits, we found that the variation coefficient of CR and CA became greater than that before the traits' subdivision (Table 1).

\section{QTL analysis of the ZS97/H94 population}

Six QTL were detected in population ZS97/H94, with three for CR and CA, respectively (Table 2). Two components traits (WC and WB) for chalkiness were further analyzed, and nine QTL (one for WCR, two for WCA, and three for WBR and WBA, respectively) were identified in this $\mathrm{DH}$ population (Table 2).

Among the QTL for CR, $q C R 5-H$ had the largest effects on chromosome 5 and explained $29.7 \%$ of phenotypic variation in Hainan and $49.3 \%$ in Wuhan. $q C R 5-H$ was also detected as a major QTL for WBR in the two environments. $q C R 6-H$ in the interval of RM435-RM170 (Wx) on chromosome 6 was also detected as a major locus. It explained $24.9 \%$ of the phenotypic variation in Hainan and $3.9 \%$ in Wuhan; $q C R 6-H$ was also the locus for CA $(q C A 6-H)$ and WBA $(q W B A 6-H)$ in Hainan. Among the QTL for CA, $q C A 1-H$ was the largest, explaining $41.3 \%$ of phenotypic variation. After integrating the QTL, the total number of QTL decreased from 15 to 12 (Table 2).

The QTL sharing frequency were rather low in the two locations (Wuhan and Hainan). Only 4 out of 15 QTL were found in the two locations, and these were the CR or WB type. None of the QTL for CA and WCR were common to both locations at a high level (Table 2).

\section{QTL analysis of the ZS97/DL208 population}

Fifteen QTL (two for CR, five each for WCR and WBA, and one each for CA, WBR, and WCA) controlling chalkiness traits were detected in population ZS97/DL308 (Table 3). qCR9-D had the largest effect for CR, explaining 
Table 1 Descriptive statistics of the traits in parents and the populations observed in Hainan and Wuhan

\begin{tabular}{|c|c|c|c|c|c|c|c|c|c|c|c|c|c|c|c|c|c|}
\hline \multirow{2}{*}{ Traits $^{a}$} & \multirow{2}{*}{ Local $^{\mathbf{b}}$} & \multicolumn{4}{|c|}{ Parents } & \multicolumn{3}{|c|}{ ZS97/H94 population } & \multicolumn{3}{|c|}{ ZS97/DL208 population } & \multicolumn{3}{|c|}{ ZS97/NYZ population } & \multicolumn{3}{|c|}{ ZS97/WYJ population } \\
\hline & & ZS97 & H94 & NYZ & WYJ & Mean & Range & $\mathrm{CV}^{\mathrm{c}} \%$ & Mean & Range & $\mathrm{CV}^{\mathrm{C}} \%$ & Mean & Range & $\mathrm{CV}^{\mathrm{c}} \%$ & Mean & Range & $\mathrm{CV}^{\mathrm{c}} \%$ \\
\hline \multirow[t]{2}{*}{ CR (\%) } & $\mathrm{H}$ & 98.0 & 10.2 & 100.0 & - & 68.4 & $0.0-100.0$ & 48.2 & 35.9 & $2.1-100.0$ & 70.5 & 93.4 & $30.3-100.0$ & 13.5 & - & - & - \\
\hline & W & 94.5 & 6.3 & 100.0 & 85.5 & 44.4 & $2.0-100.0$ & 76.8 & 23.8 & $0.0-90.0$ & 78.7 & 94.5 & $31.7-100.0$ & 11.3 & 83.2 & $10.0-100.0$ & 27.4 \\
\hline \multirow[t]{2}{*}{ CA (\%) } & $\mathrm{H}$ & 30.3 & 17.5 & 75.3 & - & 28.3 & $0.0-71.5$ & 52.1 & 22.5 & $5.5-66.0$ & 47.3 & 37.7 & $8.8-75.6$ & 37.4 & - & - & - \\
\hline & W & 28.0 & 17.3 & 78.5 & 30.5 & 31.7 & $10.2-70.1$ & 41.8 & 19.9 & $0.0-59.2$ & 61.5 & 39.8 & $8.8-86.0$ & 41.4 & 32.0 & $0.0-83.5$ & 44.1 \\
\hline \multirow[t]{2}{*}{ WCR (\%) } & $\mathrm{H}$ & 14.0 & 6.0 & 100.0 & - & 8.8 & $0.0-100.0$ & 238.8 & 24.3 & $0.0-100.0$ & 76.5 & 58.7 & $0.0-100.0$ & 68.7 & - & - & - \\
\hline & W & 30.0 & 5.0 & 100.0 & 47.0 & 7.4 & $0.0-95.0$ & 192.5 & 22.1 & $0.0-90.0$ & 87.0 & 61.8 & $0.0-100.0$ & 62.4 & 43.1 & $0.0-100.0$ & 76.0 \\
\hline \multirow[t]{2}{*}{ WBR (\%) } & $\mathrm{H}$ & 97.0 & 4.0 & 100.0 & - & 59.9 & $0.0-100.0$ & 66.3 & 12.1 & $0.0-93.7$ & 170.3 & 64.4 & $0.0-100.0$ & 64.0 & - & - & - \\
\hline & W & 85.5 & 1.0 & 100.0 & 70.0 & 36.8 & $0.0-100.0$ & 101.4 & 4.6 & $0.0-47.0$ & 192.6 & 60.6 & $0.0-100.0$ & 65.8 & 62.1 & $0.0-100.0$ & 53.6 \\
\hline \multirow[t]{2}{*}{ WCA (\%) } & $\mathrm{H}$ & 15.0 & 15.0 & - & - & 11.1 & $0.0-80.0$ & 184.4 & 23.2 & $0.0-52.5$ & 45.0 & - & - & - & - & - & - \\
\hline & W & 14.6 & 15.0 & - & 20.0 & 15.1 & $0.0-60.0$ & 128.8 & 21.2 & $0.0-59.2$ & 57.8 & - & - & - & 15.2 & $0.0-45.0$ & 71.1 \\
\hline \multirow[t]{2}{*}{ WBA (\%) } & $\mathrm{H}$ & 18.0 & 10.0 & - & - & 19.2 & $0.0-65.0$ & 74.1 & 3.9 & $0.0-23.5$ & 141.6 & - & - & - & - & - & - \\
\hline & W & 18.3 & 10.0 & - & 16.0 & 14.6 & $0.0-54.0$ & 95.4 & 6.0 & $0.0-40.0$ & 158.7 & - & - & - & 16.5 & $0.0-40.0$ & 60.0 \\
\hline
\end{tabular}

${ }^{a} C R$, chalkiness rate; $C A$, chalkiness area; $W C R$, white core rate; $W B R$, white belly rate; $W C A$, white core area; WBA, white belly area.

${ }^{\mathrm{b}} \mathrm{H}$, Hainan; W, Wuhan.

${ }^{c} \mathrm{CV}$, coefficient of variation. 
Table 2 QTL detected for chalkiness traits in population ZS97/H94

\begin{tabular}{|c|c|c|c|c|c|c|c|c|c|}
\hline \multirow{2}{*}{ Traits $^{\mathrm{a}}$} & \multirow{2}{*}{$\mathrm{Chr}^{\mathrm{b}}$} & \multirow{2}{*}{ Interval } & \multirow{2}{*}{ QTL } & \multicolumn{3}{|c|}{ Hainan } & \multicolumn{3}{|c|}{ Wuhan } \\
\hline & & & & LOD & Add $^{c}$ & $\% \operatorname{Var}^{\mathrm{d}}$ & LOD & Add $^{c}$ & $\% \operatorname{Var}^{\mathrm{d}}$ \\
\hline \multicolumn{10}{|l|}{$C R$} \\
\hline & 5 & RM574-MRG0089 & qCR5-H+ & 15.9 & 17.99 & 29.7 & 21 & 23.92 & 49.3 \\
\hline & 6 & RM435-RM170(wx) & qCR6-H+ & 14.6 & 16.47 & 24.9 & 3 & 6.7 & 3.9 \\
\hline & 12 & MRG2483-RM20A & qCR12-H+ & & & & 2.7 & 6.56 & 3.7 \\
\hline \multicolumn{10}{|l|}{ CA } \\
\hline & 1 & RM577-RM23 & qCA1-H+ & & & & 12.6 & 8.6 & 41.3 \\
\hline & 6 & RM170(wx)-RM589 & qCA6-H+ & 4.9 & 4.96 & 11.3 & & & \\
\hline & 9 & RM278-RM553 & qCA9-H+ & 8 & 6.98 & 22.4 & & & \\
\hline \multicolumn{10}{|l|}{ WCR } \\
\hline & 8 & RM310-RM126 & qWCR8-H- & & & & 5.3 & -5.97 & 17.2 \\
\hline \multicolumn{10}{|l|}{ WBR } \\
\hline & 3 & MRG2538-RM426 & qWBR3-H- & 2.3 & -6.94 & 3.1 & & & \\
\hline & 5 & MRG0089-RM289 & qWBR5-H+ & 12.3 & 20.62 & 27 & 11.1 & 20.93 & 31.5 \\
\hline & 8 & RM210-RM483 & qWBR8-H+ & 11.5 & 17.71 & 19.9 & 5.2 & 14.49 & 15.1 \\
\hline \multicolumn{10}{|l|}{ WCA } \\
\hline & 8 & RM483-RM339 & qWCA8-H- & & & & 6 & -6.43 & 10.9 \\
\hline & 9 & RM160-RM328 & qWCA9-H+ & 8.4 & 9.07 & 21.6 & & & \\
\hline \multicolumn{10}{|l|}{ WBA } \\
\hline & 5 & MRG0089-RM289 & qWBA5-H+ & & & & 4.3 & 4.04 & 8.4 \\
\hline & 6 & RM589-MX21 (wx) & qWBA6-H+ & 4.7 & 5.9 & 16.9 & & & \\
\hline & 12 & RM20A-RM179 & qWBA12-H+ & & & & 9.8 & 6.68 & 22.9 \\
\hline
\end{tabular}

${ }^{a} C R$, chalkiness rate; $C A$, chalkiness area; WCR, white core rate; WBR, white belly rate; WCA, white core area; WBA, white belly area.

${ }^{\mathrm{b}} \mathrm{Chr}$, chromosome.

'The additive (Add) effects caused by QTL; the positive value indicates that the ZS97 allele increase the trait score, while the negative value indicates that the ZS97 allele decrease the trait score.

${ }^{\mathrm{d}}$ The phenotypic variation (Var) explained by QTL.

$24.2 \%$ of the phenotypic variation on chromosome 9 , but it was only detected in Hainan. In the same locus, a QTL ( $q$ WBR9-D) was also detected for WBR in Wuhan. Both QTL could be integrated as one corresponding to WBR, with the negative additive effects of the allele from ZS97 in both environments (Hainan and Wuhan).

In Hainan, $99.2 \%$ of the whole phenotypic variation of WCA could be explained by one predominant QTL (qWCR6-D). However, this predominant QTL was replaced by three other QTL in Wuhan. For WCA, only one QTL ( $q$ WCA7-1D) was found in Wuhan, whereas none was detected in Hainan. For WBA, four QTL were found in Hainan and explained $65.1 \%$ of the phenotypic variation, whereas only one was detected in Wuhan. After integrating the QTL data, only one QTL ( $q C R 9-D$ on chromosome 9) was shared in the two locations.

\section{QTL analysis of the ZS97/NYZ population}

The chalkiness traits including CR and CA were analyzed in the population ZS97/NYZ, and 10 QTL were detected, two for CR and eight for CA (Table 4). When the two component traits WC and WB were analyzed separately, 14 QTL for chalkiness traits were found: two for CR, eight for CA, one for WCR, and three for WBR (Table 4). Interestingly, two QTL for CR on chromosome 6 could be integrated as one since both QTL had the same SSR marker (RM541). $q C R 6-N$ was also found to correspond to the loci for CA and WCR ( $q C A 6-3 N$ and $q W C R 6-N)$.

The frequency of the QTL sharing was low in the two locations in this population. Only three QTL were common to the two locations, two for WBR and one for CR. No QTL for CA was shared in the two locations at a high level, although eight QTL were detected for this trait.

\section{QTL analysis of the ZS97/WYJ population}

Seven QTL including CR and CA were detected in population ZS97/WYJ, four for CR and three for CA (Table 5). When two component traits (WC and WB) for chalkiness were further analyzed, a total of 19 QTL were detected: seven for WCR, two for WCA, six for WBR, and four for WBA (Table 5). 
Table 3 QTL detected for chalkiness traits in population ZS97/DL208

\begin{tabular}{|c|c|c|c|c|c|c|c|c|c|c|c|c|}
\hline \multirow{2}{*}{ Trait $^{a}$} & \multirow{2}{*}{$\mathrm{Chr}^{\mathrm{b}}$} & \multirow{2}{*}{ Interval } & \multirow{2}{*}{ QTL } & \multicolumn{3}{|c|}{ Hainan } & \multicolumn{3}{|c|}{ Wuhan } & \multicolumn{3}{|c|}{ Wuhan $^{\mathrm{e}}$} \\
\hline & & & & LOD & Add $^{c}$ & $\% \operatorname{Var}^{\mathrm{d}}$ & LOD & Add $^{c}$ & $\% \operatorname{Var}^{\mathrm{d}}$ & LOD & Add $^{c}$ & $\% \operatorname{Var}^{\mathrm{d}}$ \\
\hline \multicolumn{13}{|l|}{$C R$} \\
\hline & 5 & MRG5972-RM480 & qCR5-D+ & 3.2 & 11.07 & 16.3 & & & & & & \\
\hline & 9 & RM159-RM524 & qCR9-D- & 2.9 & -13.49 & 24.2 & & & & & & \\
\hline \multicolumn{13}{|l|}{ CA } \\
\hline & 6 & RM276-RM549 & qCA6-D+ & 3.1 & 3.68 & 11.6 & & & & & & \\
\hline \multicolumn{13}{|l|}{ WCR } \\
\hline & 1 & MRG5464-MRG2148 & qWCRI-D- & & & & 3.8 & -6.67 & 11.8 & 2.6 & -5.65 & 9.2 \\
\hline & 3 & RM203-RM422 & qWCR3-D- & & & & 4.1 & -6.46 & 11.1 & 2.7 & -6.78 & 13.3 \\
\hline & 6 & MX21-RM585 & qWCR6-D- & 2.7 & -19.08 & 99.2 & & & & & & \\
\hline & 7 & RM445-RM418 & $q W C R 7-D+$ & & & & 5.3 & 7.25 & 14.0 & 5.8 & 8.30 & 19.9 \\
\hline & 12 & RM235-RM17 & qWCR12-D- & & & & & & & 2.2 & -5.35 & 8.3 \\
\hline \multicolumn{13}{|l|}{ WBR } \\
\hline & 9 & RM159-RM524 & qWBR9-D- & 4.5 & -6.72 & 36.4 & & & & & & \\
\hline \multicolumn{13}{|l|}{ WCA } \\
\hline & 7 & RM478-MRG4499 & qWCA7-D+ & & & & 2.7 & 4.49 & 15.4 & 2.0 & 4.84 & 19.0 \\
\hline \multicolumn{13}{|l|}{ WBA } \\
\hline & 3 & RM251-RM282 & qWBA3-D- & 7.4 & -3.08 & 29.6 & & & & & & \\
\hline & 5 & RM39-RM164 & qWBA5-D+ & 3.4 & 1.81 & 10.2 & & & & & & \\
\hline & 8 & RM433-RM447 & qWBA8-D+ & 4.9 & 2.44 & 18.5 & & & & & & \\
\hline & 11 & RM286-RM20B & qWBA11-D+ & & & & 2.2 & 3.48 & 12.6 & & & \\
\hline & 12 & RM235-RM17 & qWBA12-D+ & 2.7 & 1.49 & 6.9 & & & & & & \\
\hline
\end{tabular}

${ }^{a} C R$, chalkiness rate; $C A$, chalkiness area; WCR, white core rate; WBR, white belly rate; WCA, white core area; WBA, white belly area

${ }^{\mathrm{b}} \mathrm{Chr}$, chromosome.

'The additive effects caused by QTL; the positive value indicates that the Zhenshan 97 allele increase the trait score, while the negative value indicates that the

Zhenshan 97 allele decrease the trait score.

d The phenotypic variation explained by QTL.

${ }^{\mathrm{e}}$ Repeat of the previous year's results in Wuhan.

Typically multiple QTL controlled the chalkiness traits, and Table 5 shows that the alleles of positive or negative effects (increasing or decreasing trait value) were dispersed in the two parents, with positive alleles at one or more loci and negative alleles at another locus. These dispersed alleles showed an overdominant phenomenon in population ZS97/WYJ. Similar phenotypes could be found in the two parents, and many QTL were detected in one location. In QTL mapping, however, phenotypic variation of the two parents was not detected.

The QTL comprehensive analysis using five populations Seventy QTL controlling chalkiness traits (including CR, CA, WCR, WCA, WBR, and WBA) were detected on all 11 chromosomes (except chromosome 10) in the four populations (ZS97/NYZ, ZS97/DL208, ZS97/H94, ZS97/ WYJ). In addition, nine QTL controlling chalkiness traits had previously been detected in the population ZS97/ MH63 [16]. Therefore, there were 79 QTL affecting chalkiness in five populations with six traits distributed in 36 distinct locations. The comprehensive QTL in the five populations were analyzed by the order of the chromosomes in rice (Additional file 1: Table S1).

On chromosome 1, nine QTL were detected in the five populations, and five loci were residual after integrating the QTL. There were two QTL in the first locus located on the short arm of chromosome 1 in the interval of MRG5464-MRG2148 (population ZS97/DL208) and that of C161-R753 (population ZS97/MH63), respectively. Thus, overlapping QTL existed, and both of the QTL were detected in Wuhan. This locus controlled WCR in population ZS97/DL208, with WCR decreasing if the allele was derived from ZS97. However, this locus controlled CR in population ZS97/MH63. The second locus also had two QTL, and both QTL in the RM84-RM283 interval controlled WBR and WBA. The locus contained three overlapping QTL controlling WBR, CA, and WCA, respectively.

On chromosome 2, two overlapping QTL controlling CR were detected in population ZS97/NYZ and ZS97/ WYJ, respectively. The alleles from ZS97 were associated with increased CR, and both of the QTL belonged to one locus. 
Table 4 QTL detected for chalkiness traits in population ZS97/NYZ

\begin{tabular}{|c|c|c|c|c|c|c|c|c|c|}
\hline \multirow{2}{*}{ Trait $^{a}$} & \multirow{2}{*}{$\mathrm{Chr}^{\mathrm{b}}$} & \multirow{2}{*}{ Interval } & \multirow{2}{*}{ QTL } & \multicolumn{3}{|c|}{ Hainan } & \multicolumn{3}{|c|}{ Wuhan } \\
\hline & & & & LOD & $\operatorname{Add}^{c}$ & $\% \operatorname{Var}^{d}$ & LOD & $\operatorname{Add}^{c}$ & $\% \operatorname{Var}^{d}$ \\
\hline \multicolumn{10}{|l|}{$C R$} \\
\hline & 2 & RM183-RM526 & qCR2-N- & 3.5 & -3.33 & 6.8 & & & \\
\hline & 6 & RM527- MRG2498 & $q C R 6-1 N-$ & 5.6 & -3.65 & 8.1 & 5.0 & -3.91 & 13.4 \\
\hline \multicolumn{10}{|l|}{ CA } \\
\hline & 1 & RM488-RM246 & qCA1-N- & & & & 3.6 & -3.58 & 4.6 \\
\hline & 3 & RM545-RM517 & $9 C A 3-1 N-$ & & & & 5.6 & -5.09 & 9.2 \\
\hline & 3 & RM468-RM570 & $9 C A 3-2 N-$ & & & & 2.6 & -2.95 & 3.1 \\
\hline & 6 & RM190-RM587 & qCA6-1N- & 1.9 & -2.79 & 3.9 & & & \\
\hline & 6 & RM585-RM557 & $9 C A 6-2 N-$ & & & & 6.9 & -4.52 & 7.3 \\
\hline & 6 & MRG2498-RM454 & $9 C A 6-3 N-$ & 3.4 & -3.77 & 7.1 & & & \\
\hline & 9 & RM296-RM321 & qCA7-N- & & & & 4.8 & -4.12 & 6.0 \\
\hline & 11 & RM332-RM167 & qCA8-N- & & & & 9.1 & -5.36 & 10.2 \\
\hline \multicolumn{10}{|l|}{ WCR } \\
\hline & 6 & MRG2498-RM454 & qWCR6-N- & 2.9 & -10.35 & 6.7 & & & \\
\hline \multicolumn{10}{|l|}{ WBR } \\
\hline & 1 & RM490-RM600 & qWBR1-N+ & 3.3 & 12.61 & 9.2 & & & \\
\hline & 8 & RM264-RM477 & qWBR8-N+ & 3.0 & 11.05 & 7.1 & 5.0 & 12.32 & 9.4 \\
\hline & 12 & RM101-RM519 & qWBR12-N+ & 3.6 & 14.55 & 12.3 & 3.3 & 12.40 & 9.6 \\
\hline
\end{tabular}

${ }^{a} C R$, chalkiness rate; $C A$, chalkiness area; WCR, white core rate; WBR, white belly rate; WCA, white core area; WBA, white belly area.

${ }^{\mathrm{b}} \mathrm{Chr}$, chromosome.

'The additive effects caused by QTL; the positive value indicates that the Zhenshan 97 allele increase the trait score, while the negative value indicates that the Zhenshan 97 allele decrease the trait score.

${ }^{\mathrm{d}}$ The phenotypic variation explained by QTL.

On chromosome 3, eight QTL were detected in four populations (population ZS97/MH63 was not included). The eight QTL were interspersed at six loci, three of which contained two QTL. These two QTL were located in two overlapping intervals of RM251-RM282 and MRG2803RM282 and controlled WBA and CA in populations ZS97/ DL208 (Hainan) and ZS97/WYJ (Wuhan), respectively. There were also two QTL controlling CA and WCR, respectively, in the overlapping interval of RM468-RM570 and RM130-RM570 at the sixth loci detected in Wuhan; both QTL were also possible for two independent loci. Interestingly, the other four loci had only one QTL.

On chromosome 4, five QTL controlling WC and distributed over three loci were detected in population ZS97/ WYJ. Three QTL controlling CR, WCR, and WCA, respectively, were interspersed in the RM335-MRG5943 interval, and the chalkiness effects decreased if the alleles were derived from ZS97. Single QTL controlling WCR were detected at the second and third locus, respectively, and the additive effects were detected if the allele was derived from ZS97.

On chromosome 5, ten QTL in four populations (except population ZS97/NYZ) were detected, and only one originated from population ZS97/MH63. At the first locus, six QTL controlling CR, WBR, and WBA were detected in the interval of RM574-MRG0089-RM289 (population ZS97/H94) or RG360-C734a (population ZS97/MH63). Interestingly, the allele derived from ZS97 increased WBR while decreasing WC, and this locus was previously shown to affect grain width and WBR in population ZS97/MH63 [16]. These vital effects were found in both Wuhan and Hainan, and this phenomenon was noteworthy. At the third locus, three overlapping QTL controlling CR or WBR were detected in three populations in both Hainan and Wuhan.

On chromosome 6, fourteen QTL interspersed at three loci were detected in five populations. Two types of QTL could be divided into eight QTL at the first locus ( $W x$ locus): one type of QTL controlling WCR or CR was detected in both Wuhan and Hainan, and the other type of QTL controlling WBA and CA. The alleles from different populations had effects in various directions. Thus, there was no specific type of chalkiness traits because of the two types of QTL, although it was possible that two tightly linked loci existed. The Alk gene encoding soluble amylase (starch synthesis-related enzyme), another important gene for cooking and eating quality, was also at this locus (Additional file 1: Table S1).

On chromosome 7, five QTL were detected at three loci in three populations (Figure 1). One QTL controlling CR 
Table 5 QTL detected for chalkiness traits in population ZS97/WYJ

\begin{tabular}{|c|c|c|c|c|c|c|}
\hline \multirow{2}{*}{ Trait $^{a}$} & \multirow{2}{*}{$\mathrm{Chr}^{\mathrm{b}}$} & \multirow{2}{*}{ Interval } & \multirow{2}{*}{ QTL } & \multicolumn{3}{|c|}{ Wuhan } \\
\hline & & & & LOD & $\operatorname{Add}^{c}$ & $\% \operatorname{Var}^{\mathrm{d}}$ \\
\hline \multicolumn{7}{|l|}{$C R$} \\
\hline & 2 & RM263-RM221 & qCR2-W- & 3.3 & -6.63 & 8.7 \\
\hline & 4 & RM335-MRG5943 & qCR4-W- & 3.9 & -5.30 & 6.9 \\
\hline & 7 & RM82-RM125 & qCR7-W- & 3.4 & -6.06 & 7.3 \\
\hline & 9 & RM296-RM285 & qCR9-W+ & 3.8 & 6.28 & 7.8 \\
\hline \multicolumn{7}{|l|}{ CA } \\
\hline & 3 & MRG2803-RM282 & qCA3-W- & 5.4 & -4.15 & 8.6 \\
\hline & 6 & RM190-RM510 & qCA6-W+ & 3.9 & 3.47 & 6.0 \\
\hline & 9 & RM285-MRG6094 & qCA9-W+ & 3.6 & 3.20 & 5.1 \\
\hline \multicolumn{7}{|l|}{ WCR } \\
\hline & 3 & RM36-MRG0002 & qWCR3-1W- & 9.0 & -12.56 & 14.3 \\
\hline & 3 & RM130-RM570 & qWCR3-2W- & 3.1 & -6.90 & 4.3 \\
\hline & 4 & RM335-MRG5943 & qWCR4-1W- & 13.8 & -14.17 & 18.2 \\
\hline & 4 & RM142-RM177 & qWCR4-2W+ & 3.2 & 8.09 & 6.3 \\
\hline & 4 & RM252-RM241 & qWCR4-3W+ & 10.4 & 11.92 & 12.9 \\
\hline & 8 & RM80-RM149 & qWCR8-W- & 4.3 & -9.29 & 8.3 \\
\hline & 9 & RM285-MRG6094 & qWCR9-W+ & 8.6 & 12.40 & 13.9 \\
\hline \multicolumn{7}{|l|}{ WBR } \\
\hline & 1 & RM84-RM283 & qWBR1-1W- & 4.1 & -8.59 & 7.7 \\
\hline & 1 & RM129-RM9 & qWBR1-2W+ & 4.2 & 8.08 & 6.8 \\
\hline & 5 & RM87-RM334 & qWBR5-W+ & 2.5 & 6.09 & 3.9 \\
\hline & 8 & RM152-RM38 & qWBR8-W- & 13.3 & -14.04 & 17.6 \\
\hline & 9 & RM296-RM285 & qWBR9-W+ & 7.6 & 10.73 & 10.3 \\
\hline & 11 & RM536-RM287 & qWBR11-W- & 8.0 & -10.29 & 9.4 \\
\hline \multicolumn{7}{|l|}{ WCA } \\
\hline & 1 & RM259-RM312 & qWCA1-W+ & 3.8 & 4.01 & 13.8 \\
\hline & 4 & RM335-MRG5943 & qWCA4-W- & 4.4 & -3.88 & 12.8 \\
\hline \multicolumn{7}{|l|}{ WBA } \\
\hline & 1 & RM84-RM283 & qWBA1-W- & 2.5 & -2.64 & 7.1 \\
\hline & 7 & RM505-RM18 & qWBA7-W- & 2.0 & -2.53 & 6.5 \\
\hline & 8 & RM210-RM80 & qWBA8-W+ & 3.0 & 2.61 & 8.0 \\
\hline & 11 & RM536-RM287 & qWBA11-W- & 4.8 & -3.95 & 15.9 \\
\hline
\end{tabular}

${ }^{a} \mathrm{CR}$, chalkiness rate; $\mathrm{CA}$, chalkiness area; $\mathrm{WCR}$, white core rate; WBR, white belly rate; WCA, white core area; WBA, white belly area.

${ }^{\mathrm{b}} \mathrm{Chr}$, chromosome.

'The additive effects caused by QTL; the positive value indicates that the ZS97 allele increase the trait score, while the negative value indicates that the ZS97 allele decrease the trait score.

dThe phenotypic variation explained by QTL.

was detected at the first locus, and two QTL controlling grain WC were detected at the second locus. Two QTL controlling grain $\mathrm{WB}$ were also detected at the third locus.

On chromosome 8, eight QTL were interspersed at four loci, and four QTL at the third loci were detected in four populations (except population ZS97/MH63). Interestingly, WBR increased and WCA decreased simultaneously if the alleles were derived from ZS97 in population ZS97/H94, while WBA increased and WCR decreased simultaneously if the alleles were derived from ZS97 in population ZS97/ WYJ. All these QTL demonstrated reciprocal inhibition among different types of chalkiness traits, similar to a locus of QTL on chromosome 5. Moreover, two QTL detected at the fourth locus from ZS97 enhanced WBR or WBA.

On chromosome 9, nine QTL interspersed at three loci were detected in four populations (except population ZS97/MH63), and seven of them could be divided into two types controlling $\mathrm{CA}$ and $\mathrm{WB}$ at the first locus. Interestingly, multiple alleles were also detected, and this was similar to the $W x$ locus on chromosome 6. A QTL controlling CA or WCA was detected in the other two loci, respectively.

On chromosome 10, only one QTL controlling CR was detected [16].

On chromosome 11, four QTL were detected at three loci in population ZS97/WYJ, with two QTL at the third locus. Both WBR and WBA decreased simultaneously if the alleles were derived from ZS97.

On chromosome 12, five QTL were detected at two loci. Three QTL controlling CR, WBR, and WBA, respectively, were at the first locus. Two QTL at the second locus controlled increased WBA and decreased WCR simultaneously in population ZS97/DL208 if the alleles were derived from ZS97; this character was similar to the locus on chromosome 5 (Additional file 1: Table S1, Figure 1).

Taken together, some overlapping QTL controlled the same types of chalkiness in five populations. Therefore, we could integrate the 79 QTL into 36 loci, 21 of which clustered together and contained 64 QTL (Additional file 1: Table S2).

\section{Confirmation of the identified QTL}

There were 79 QTL affecting chalkiness traits with six chalkiness traits (CR, CA, WCR, WCA, WBR, and WBA) across two environments. These QTL were integrated into 36 distinct locations on 12 chromosomes in five populations (Figure 1 and Additional file 1: Table S1). Twenty-one of the 36 distinct locations contained 64 QTL clustered together (QTL clusters), which were distributed on 11 chromosomes (except for chromosome 10) in the five populations. With regard to the rate of chalkiness traits, 11 of 21 QTL clusters from ZS97 displayed rate increases, while the others displayed rate decreases (Additional file 1: Table S3). Interestingly, 15 of 21 QTL clusters (71.4\%) were identified in two or more populations (Additional file 1: Table S3), and the QTL cluster on chromosome 6 was detected in all five populations (Figure 1). Thus, the QTL clusters were relatively stable, and 13 of 36 distinct 
locations $(36.1 \%)$ were consistently detected in Wuhan and Hainan (Additional file 1: Table S2).

To further confirm QTL, WG97 (ZS97 genetic background, with low or no chalkiness) and ZS97 were chosen as parents to construct an $\mathrm{F}_{2}$ population (1398 individuals) in 2008 in Wuhan. One main-effect QTL $(q C R 5-H+$, the phenotypic variation explained by QTL for $49.3 \%$ and 29.7\% in Wuhan and Hainan, respectively, Table 2) controlling CR, WBR, and WBA was knocked out by marker-assisted selection from the $F_{2}$ population with two tightly linked molecular markers (RM574 and MRG0089). Consequently, 396 individuals derived from ZS97 were further analyzed by the tightly linked SSR markers (RM445-RM418, MRG5972-RM480, RM490-RM600, RM264-RM477, and RM101-RM519), respectively. The results indicated that the QTL effects could be reproduced and showed dominance ( $q$ WBR1, qWBR8, qWBR12 and $q C R 5)$ or overdominance effects $(q W C R 7)$ for the rate of the chalkiness traits in this $\mathrm{F}_{2}$ population (ZS97/WG97). Moreover, five QTL could individually explain more than $10 \%$ of the variation of the trait; more than $15 \%$ of WBR and $23 \%$ of $C R$ were explained if the gene regions were derived from ZS97 (Table 6). $q W B R 1, q W B R 8$, and $q W B R 12$ controlling WBR were identified again, and those were similar to the QTL clusters on chromosomes 1, 8, and 12, respectively (Table 6, Additional file 1: Table S3). Another QTL cluster controlling WBR was detected on chromosome 9 (Additional file 1: Table S3), and it was also found across eight environments [19]. Therefore, all four QTL clusters derived from ZS97 that controlled WB rate were stable and reliable (Additional file 1: Table S3).

\section{Discussion}

Comparative analysis of multiple QTL mapping by alignment to a common genetic map offers a more complete picture of the genetic control of a trait than can be obtained by any other approach [48]. In this study, 79 QTL controlling six chalkiness traits (CR, WCR, WBR, CA, WCA, and WBA) were detected in five populations (ZS97/H94, ZS97/ NYZ, ZS97/DL208, ZS97/WUJ, and ZS97/MH63). The
QTL were integrated into 36 loci, 21 of which were clustered together and contained 64 of the QTL. In addition, 15 of 21 (71.4\%) QTL clusters were found in two or more populations (Additional file 1: Table S3), and 13 of $36(36.1 \%)$ distinct locations were consistently detected in Wuhan and Hainan (Additional file 1: Table S2). Therefore, the QTL clusters controlling grain chalkiness are relatively stable. However, 15 QTL still occur separately on different chromosomes. Our results confirm that chalkiness traits are mainly controlled by some major QTL, and they are important to varying degrees in five populations with different genetic backgrounds. In fact, there are many QTL in four populations (ZS97/H94, ZS97/WYJ, ZS97/DL208, and ZS97/NYZ), and a new QTL (R2625-C223, on chromosome 10) increased through integration the results of population ZS97/MH63. Thus, by subdividing the chalkiness traits, it was possible to detect many more QTL and to determine that they have reciprocal conformity in multiple environments and populations with different genetic backgrounds.

\section{Chalkiness QTL common to the different environments}

The three populations (ZS97/H94, ZS97/DL208, and ZS97/ NYZ) were all planted in Wuhan and Hainan, which represented two different environments. These populations were concomitantly planted in the same fields in two environments, respectively, with consistent soil fertility and field management [26]. ZS97 was their common parent, thus our results have a strong basis for comparison and reliability because it has been shown that some characters can be affected by different planting years and environments [19]. Seventy-nine QTL controlling chalkiness traits were integrated into 36 distinct locations on 12 chromosomes, and 13 of the 36 (36.1\%) distinct locations were consistently detected in Wuhan and Hainan (Additional file 1: Table S2, Figure 1). The degree of correlation between the shared extent and characters of the QTL was consistent in the two environments, with the extent of sharing based on population being ZS97/H94 > ZS97/NYZ > ZS97/DL208 (Additional file 1: Table S1).

Table 6 Validation of 5 QTL in $F_{2}$ population ZS97/WG97

\begin{tabular}{|c|c|c|c|c|c|c|c|c|c|c|c|c|c|c|c|c|}
\hline \multirow{2}{*}{ Trait $^{\mathrm{a}}$} & \multirow{2}{*}{$\mathrm{Chr}^{\mathrm{b}}$} & \multirow{2}{*}{ Interval } & \multirow{2}{*}{ QTL } & \multicolumn{4}{|c|}{ Wuhan 2008} & \multicolumn{3}{|c|}{ Hainan 2004} & \multicolumn{3}{|c|}{ Wuhan 2004} & \multicolumn{3}{|c|}{ Wuhan 2002} \\
\hline & & & & LOD & Add $^{c}$ & $\operatorname{Dom}^{d}$ & $\% \operatorname{Var}^{\mathrm{e}}$ & LOD & Add $^{c}$ & $\% \operatorname{Var}^{\mathrm{e}}$ & LOD & Add $^{c}$ & $\% \operatorname{Var}^{\mathrm{e}}$ & LOD & Add $^{c}$ & $\% \operatorname{Var}^{\mathrm{e}}$ \\
\hline WBR & 8 & RM264-RM477 & qWBR8 & 4.6 & 6.30 & 8.89 & 12.5 & 3.0 & 11.05 & 7.1 & 5.0 & 12.32 & 9.4 & & & \\
\hline WBR & 12 & RM101-RM519 & qWBR12 & 7.7 & 10.53 & 11.53 & 16.1 & 3.6 & 14.55 & 12.3 & 3.3 & 12.40 & 9.6 & & & \\
\hline WCR & 7 & RM445-RM418 & qWCR7 & 4.2 & 10.81 & 23.12 & 12.5 & & & & 5.3 & 7.25 & 14.0 & 5.8 & 8.30 & 19.9 \\
\hline WBR & 1 & RM490-RM600 & qWBR1 & 3.8 & 12.68 & 17.21 & 10.2 & 3.3 & 12.61 & 9.2 & & & & & & \\
\hline$C R$ & 5 & MRG5972-RM480 & qCR5 & 7.7 & 12.18 & 14.68 & 16.4 & 3.2 & 11.07 & 16.3 & & & & & & \\
\hline
\end{tabular}

${ }^{\mathrm{a}} \mathrm{WBR}$, white belly rate; $\mathrm{CR}$, chalkiness rate.

${ }^{\mathrm{b}} \mathrm{Chr}$, chromosome.

'The additive effects caused by QTL, the positive value indicates that the ZS97 allele increase the trait score.

${ }^{\mathrm{d}}$ Dom, dominant effects.

${ }^{\mathrm{e}}$ The phenotypic variation explained by QTL. 
The correlation coefficients between WBR and CR were higher than for the other traits in the two environments (Additional file 1: Table S4). Hence, the QTL associated with these traits were detected in the environment more frequently than QTL associated with other chalkiness traits. The reactions of the different populations in the same environment are also extraordinarily different [39], and chalkiness traits are affected by the weather conditions especially during the grain filling period [24]. However, the QTL identified in one environment did not necessarily yield the same results in another environment, possibly due to experimental errors or different test thresholds.

\section{Chalkiness QTL shared by different populations}

After comparing the five populations in Wuhan and three populations in Hainan, we found few shared QTL for populations ZS97/H94, ZS97/NYZ, and ZS97/DL208, while populations ZS97/DL208, ZS97/WYJ, and ZS97/MH63 contained many of the same QTL. Fifteen of 21 (71.4\%) QTL clusters were identified in two or more populations (Additional file 1: Table S3), and the QTL cluster on chromosome 6 was detected in five populations (Figure 1). Further research revealed that some QTL having minor effects in one population had major effects in other populations. On chromosome 1, one QTL controlling grain width was detected in population ZS97/DL208 and ZS97/ MH63, and the effects were the same as chalkiness traits. Meanwhile, many QTL were mutual in population ZS97/ H94 and ZS97/MH63, which were all low-chalkiness grains. Interestingly, our results are similar to earlier findings suggesting that the extent of QTL shared in populations is related to the genetic diversity of the different parents [15,49]; therefore, the six parents used in this were also analyzed (Figure 2).

\section{The relationship between chalkiness traits and the QTL}

Through QTL analysis, the 79 QTL can be divided into three types. The first type has no specificity for chalkiness traits and only affects chalkiness such as the $W x$ locus on chromosome 6; this locus affects the different chalkiness types in various environments, populations, and even in the same population (Additional file 1: Table S1). The second type only has specificity for chalkiness traits without specificity for grain CR or CA. However, the third type controls only a single grain chalkiness trait and has the strongest specificity (Additional file 1: Table S1).

According to the relationship between chalkiness traits and the QTL, another three types are apparent in this study. The first type is "independent," meaning that the QTL are independent from each other and a chalkiness trait is only affected by one specific QTL. The second type is "symbiosis," which involves various chalkiness traits being controlled by a locus and showing an effect in the same direction. The third type is "competition" in which the QTL increases one chalkiness trait but simultaneously decreases other phenotypes (e.g., the QTL on chromosome 8) (Additional file 1: Table S1, Figure 1). Therefore, our results are similar to findings suggesting a complex relationship between chalkiness traits and the QTL. While chalkiness is under genetic control [16,50], the traits differ from each other with regard to their genetic mechanisms, but sometimes they also contact with each other [24]. Our results showed that the chalkiness traits were also under genetic control, the environmental impact was relatively weak, and dominance and overdominance effects were the key factors affecting grain chalkiness in rice.

\section{Benefits of multiple populations, multiple environments, and chalkiness traits}

In this study, a large number of QTL controlling chalkiness traits in rice grain were detected, and the use of five populations, two environments, and six chalkiness traits will contribute to a more complete understanding of the genetic mechanism of the chalkiness traits. Moreover, the characterization of the QTL can be done more accurately if multiple environments and chalkiness traits are analyzed in multiple populations because of the low heritability of chalkiness traits $[19,24,51]$. Although some instrumentation exists for measuring grain chalkiness, grain

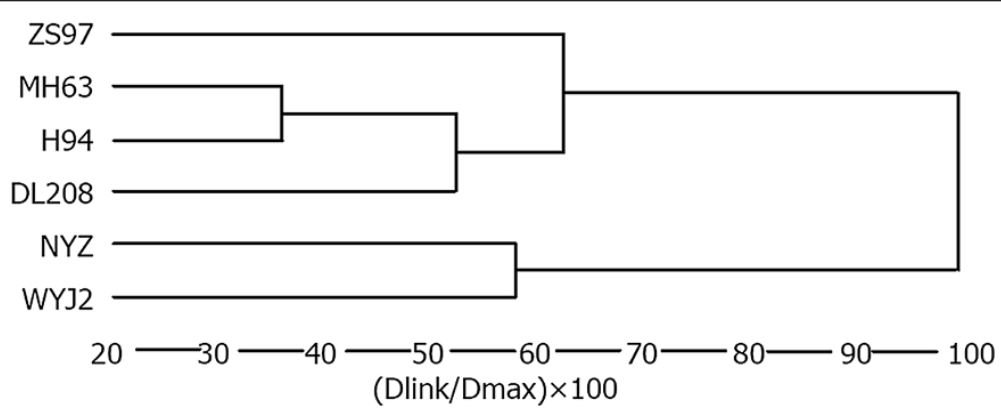

Figure 2 Cluster dendrogram of six parents used in this study as resolved by complete linkage using molecular marker difference as the distance measure. 
WB cannot be distinguished from other chalkiness traits in this manner and the phenotypes could obscure the chalkiness QTL effects; therefore, we analyzed the chalkiness traits by visual inspection despite some difficulties. In addition, many QTL may be overlooked if the highstandard LOD value is adopted, while many more QTL may be discovered if the low-standard LOD value is used in analysis. Therefore, we used multiple populations, multiple environments, and chalkiness traits to prove the QTL reciprocally. Finally, multiple-population and multiple-environment analysis in our study is beneficial for investigating the different genetic mechanism of the chalkiness traits, the relationships among the chalkiness traits and the various manifestations in different environments, and populations with different genetic backgrounds. Interestingly, all of the previously reported loci were detected in our study except for a weak effect QTL (R2625-C223) located on chromosome 10 [16,19,50,52]. These results essentially satisfied our expectations that as many QTL as possible would be detected within multiple environments and for multiple traits; further, the results in one environment confirmed those in other environments. In addition, ZS97 is the common parent in five populations and is also a parent of Shanyou 63, which is the most widely grown rice hybrid in China. Consequently, our results have great significance for improving hybrid rice, which is widely planted in more than 20 countries around the world.

\section{The interpretation for chalkiness QTL and chalkiness formation mechanism}

The chalkiness traits under genetic control involve many QTL [16,19,50,52]. These chalkiness traits are susceptible to environmental conditions and management practice under field conditions, and a considerable proportion of the traits are simultaneously caused by dominant or overdominant effects (Table 6). All the features considered in the current study commonly occurred in QTL analyses of yield traits in the literature [52,53]. Therefore, chalkiness trait occurrence is obviously a complex process, and it might be affected by the relationships among the source, storeroom, and streams [54]. Moreover, any change in these three factors or lack of coordination among them would influence or engender chalkiness in the endosperm. The modes of action and approaches for QTL were analyzed based on speculations about the three chalkiness traits. The utilization of QTL was a powerful means to estimate gene action of QTL and to conduct fine mapping of QTL to dissect complex rice endosperm chalkiness [55]. Our research also showed that some of the genes related to starch synthesis, such as $W x$ and $A l k$, might play an important role in the formation of chalkiness. In addition, various types of chalkiness QTL might be related to different chalkiness structure characteristics, filling periods, filling temperatures, and filling dynamics; for example, alpha-amylase has functional roles in the development of a chalky endosperm [56].

Since the chalkiness QTL effects can obscure each other's phenotype, one main-effect QTL $(q C R 5-H+)$ controlling CR, WBR, and WBA was knocked out by marker-assisted selection from an $F_{2}$ population. Our results showed that all four QTL clusters derived from ZS97 controlling the rate of chalkiness can be stably and reliably detected (Additional file 1: Table S3), and the other QTL are more critical in heterozygous individuals than the loci derived from ZS97 and show transgressive segregation in the $F_{2}$ population (Additional file 1: Table S3). In addition, it was previously reported that the QTL on chromosome 5 controlling grain WB also controlled grain width, which is closely associated with chalkiness [16,19]. Moreover, the flo4 gene controlling WC endosperm on chromosome 5 was also detected in the MRG5972-RM480 interval in this study [57]. For that reason, the effects of QTL in our comparative map are stable, although the other QTL need further confirmation. In rice breeding, some QTL derived from the alleles (non-ZS97), such as $q W B R 3-H$ (Table 2) and $q$ WCR6-D (Table 3), decrease chalkiness traits. In theory, if all of these QTL were polymerized together for ZS97, grains (chalkiness-free) could be produced in diverse environments. Compared with the previous QTL, our results showed that the QTL clusters derived from ZS97 are stably and reliably identified in different populations and environments (Additional file 1: Table S3). Our comparison map includes all previous QTL of chalkiness traits, and most of the QTL in this study are unified and integrated.

\section{Conclusions}

Our study identified 79 QTL associated with six chalkiness traits using five populations (two DH lines and three RILs) across two environments and obtained an integrated genetic map, which included 430 markers. The QTL were distributed across 36 distinct locations, and $58.3 \%$ of locations clustered together. In addition, $71.4 \%$ of the QTL clusters were identified in two or more populations, and $36.1 \%$ of QTL were consistently detected in two different environments. Results from this study facilitate our understanding of the QTL related to chalkiness traits in different populations and various environments and the genetic basis. Interestingly, the QTL could be detected in different populations, and four QTL clusters derived from ZS97 controlling WBR were stably and reliably detected in the $F_{2}$ population, indicating that the identified QTL clusters are consistent in different populations and environments. Thus, our results have paved the way for rice breeding by marker-assisted selection and map-based cloning of important QTL or genes, as well as the genetic improvement of grain quality in rice and potentially other staple crops. 


\section{Additional file}

Additional file 1: Table S1. Integration of the QTL for chalkiness traits in five populations. Table S2 Number of QTL for chalkiness traits in five populations. Table S3 Information of the QTL clusters for the chalkiness traits in five populations. Table S4 Coefficients of pairwise correlations of the same chalkiness trait between two environments in three populations.

\section{Abbreviations}

QTL: Quantitative trait loci; DH: Doubled haploid; RILs: Recombinant inbred lines; DNA: Deoxyribonucleic acid; ZS97: Zhenshan 97; DL208: Delong 208; NYZ: Nanyangzhan; WYJ: Wuyujing; MH63: Minghui 63; CR: Chalkiness rate; CA: Chalkiness area; WBR: White belly rate; WBA: White belly area; WCR: White core rate; WCA: White core area; SSR: Simple sequence repeat; LR: Likelihood ratio; WB: White core; WC: White belly; H: Hainan; W: Wuhan.

\section{Competing interests}

The authors declare they have no competing interests.

\section{Authors' contributions}

$\mathrm{BP}$ and LQW were involved in the conception of the experiment, analysis, interpretation of the data, and drafting the article. CCF, GHJ, LJL, and YBL contributed to the interpretation of the results and helped to draft the manuscript. YQH designed the experiment, did quality control for the data, and performed the analysis. All authors read, contributed, and approved the manuscript.

\section{Acknowledgements}

This work was supported by grants from the National 863 Project (2012AA10A303), the National Program on R\&D of Transgenic Plants (2013ZX08009003-004), the National Natural Science Foundation (31171523), Foundation of Ministry of (CARS-01-03, 2012G2) in China.

\section{Author details}

${ }^{1}$ National Key Laboratory of Crop Genetic Improvement, National Center of Plant Gene Research and National Center of Crop Molecular Breeding, Huazhong Agricultural University, Wuhan 430070, China. ${ }^{2}$ Shanghai Agrobiological Gene Center, Shanghai 201106, China.

Received: 19 January 2014 Accepted: 16 April 2014

Published: 26 April 2014

\section{References}

1. Fitzgerald MA, McCouch SR, Hall RD: Not just a grain of rice: the quest for quality. Trends Plant Sci 2009, 14:133-139.

2. Cheng SH, Zhuang JY, Fan YY, Du JH, Cao LY: Progress in research and development on hybrid rice: a super-domesticate in China. Ann Bot 2007, 100:959-966.

3. Zhang QF: Strategies for developing green super rice. Proc Natl Acad Sci US A 2007, 104:16402-16409.

4. Wan XY, Weng JF, Zhai HQ, Wang JK, Lei CL, Liu XL, Guo T, Jiang L, Su N, Wan JM: Quantitative trait loci (QTL) analysis for rice grain width and fine mapping of an identified QTL allele gw-5 in a recombination hotspot region on Chromosome 5. Genetics 2008, 179:2239-2252.

5. Yoshioka Y, Iwata H, Tabata M, Ninomiya S, Ohsawa R: Chalkiness in rice: potential for evaluation with image analysis. Crop Sci 2007, 47:2113-2120.

6. Shen XP, Shen XY, Li G, Gon GP, Zhang HC: Effect of seeding time on chalkiness of liangyoupeijiu in Jiangsu rice growing areas at different latitudes. Chinese J Rice Sci 2007, 21:677-680.

7. Tin $H Q$, Berg T, Bjørnstad $\AA$ : Diversity and adaptation in rice varieties under static (ex situ) and dynamic (in situ) management. Euphytica 2001, 122:491-502.

8. Cheng FM, Zhong LJ, Wang F, Zhang GP: Differences in cooking and eating properties between chalky and translucent parts in rice grains. Food Chem 2005, 90:39-46.

9. Wang JK, Wan XY, Li HH, Pfeiffer WH, Crouch J, Wan JM: Application of identified QTL-marker associations in rice quality improvement through a design-breeding approach. Theor Appl Genet 2007, 115:87-100.

10. Liu X, Wan X, Ma X, Wan J: Dissecting the genetic basis for the effect of rice chalkiness, amylose content, protein content, and rapid viscosity analyzer profile characteristics on the eating quality of cooked rice using the chromosome segment substitution line population across eight environments. Genome 2011, 54:64-80.

11. Zheng L, Zhang W, Liu S, Chen L, Liu X, Chen X, Ma J, Chen W, Zhao Z, Jiang $L$, Wan J: Genetic relationship between grain chalkiness, protein content, and paste viscosity properties in a backcross inbred population of rice. J Cereal Sci 2012, 56:153-160.

12. Tsukaguchi $T$, lida $Y$ : Effects of assimilate supply and high temperature during grain-filling period on the occurrence of various types of chalky kernel in rice plants (Oryza sativa L.). Plant Prod Sci 2008, 11:203-210

13. Takeda K, Saito K: Heritability and genetic correlation of kernel weight and white belly frequency in rice. Japan J Breed 1983, 33:468-480.

14. Peng S, Huang J, Sheehy JE, Laza RC, Visperas RM, Zhong X, Centeno GS, Khush GS, Cassman KG: Rice yields decline with higher night temperature from global warming. Proc Natl Acad Sci U S A 2004, 101:9971-9975.

15. He YQ, Yang J, Xu CG, Zhang ZG, Zhang QF: Genetic bases of instability of male sterility and fertility reversibility in photoperiod-sensitive genic male-sterile rice. Theor Appl Genet 1999, 99:683-693.

16. Tan YF, Xing YZ, Zhang QF, Li JX, Yu SB: Genetic bases of appearance quality of rice grains in Shanyou 63, an elite rice hybrid. Theor App/ Genet 2000, 101:823-829.

17. Yoshida S, Ikegami M, Kuze J, Sawada K, Hashimoto Z, Ishii T, Nakamura C, Kamijima O: QTL analysis for plant and grain characters of sake-brewing rice using a doubled haploid population. Breed Sci 2002, 52:309-317.

18. Li ZF, Wan JM, Xia JF, Zhai HQ: Mapping quantitative trait loci underlying appearance quality of rice grains (Oryza sativa L.). Acta Genet $\operatorname{Sin} 2003$, 30:251-259.

19. Wan XY, Wan JM, Weng JF, Jiang L, Bi JC, Wang CM, Zhai HQ: Stability of QTLs for rice grain dimension and endosperm chalkiness characteristics across eight environments. Theor Appl Genet 2005, 110:1334-1346.

20. Mei DY, Zhu YJ, Yu YH, Fan YY, Huang DR, Zhuang JY: Quantitative trait loci for grain chalkiness and endosperm transparency detected in three recombinant inbred line populations of indica rice. J Int Agric 2013, 12:1-11.

21. Yamakawa H, Ebitani T, Terao T: Comparison between locations of QTLs for grain chalkiness and genes responsive to high temperature during grain filling on the rice chromosome map. Breed Sci 2008 58:337-343.

22. Ohsawa R, lida Y, Hirabayashi H, Takeuchi Y, Tabata M, Ando I: Mapping of quantitative trait loci for the occurrence of white-back kernels associated with high temperatures during the ripening period of rice (Oryza sativa L.). Breed Sci 2007, 57:47-52.

23. Kobayashi A, Genliang B, Shenghai Y, Tomita K: Detection of quantitative trait loci for white-back and basal-white kernels under high temperature stress in japonica rice varieties. Breed Sci 2007, 57:107-116.

24. Yamakawa H, Hirose $T$, Kuroda M, Yamaguchi T: Comprehensive expression profiling of rice grain filling-related genes under high temperature using DNA microarray. Plant Physiol 2007, 144:258-277.

25. Kepiro JL, McClung AM, Chen MH, Yeater KM, Fjellstrom RG: Mapping QTLs for milling yield and grain characteristics in a tropical japonica long grain cross. J Cer Sci 2008, 48:477-485.

26. Yang JC, Chang EH, Tang C, Zhang H, Wang ZQ: Relationships of ethylene evolution rate and 1-aminocylopropane-1-carboxylic acid concentration in grains during filling period with appearance quality of rice. Rice Sci 2007, 14:33-41.

27. Koh HJ, Heu MH: Agronomic characteristics of a mutant for genic male sterility-chalky endosperm and its utilization on $\mathrm{F}_{1}$ hybrid breeding system in rice. Korean J Crop Sci 1995, 40:684-696.

28. Koh HJ, Son YH, Heu MH, Lee HS, McCouch SR: Molecular mapping of a new genic male-sterility gene causing chalky endosperm in rice (Oryza sativa L.). Euphytica 1999, 106:57-62.

29. Woo MO, Ham TH, Ji HS: Inactivation of the UGPase1 gene causes genic male sterility and endosperm chalkiness in rice (Oryza sativa L.). Plant J 2008, 54:190-204.

30. Kao GH, Zeng ZB: General formulas for obtaining the MLEs and asymptotic variance-covariance matrix mapping quantitative trait loci when using the EM algorithm. Biometrics 1997, 53:653-665.

31. Thabuis A, Palloix A, Pflieger S, Daubze AM, Caranta C, Lefebvre V: Comparative mapping of Phytophthora resistance loci in pepper germplasm: evidence for conserved resistance loci across Solanaceae and for a large genetic diversity. Theor App/ Genet 2003, 106:1473-1485. 
32. Chen $\mathrm{S}$, Lin XH, Xu CG, Zhang QF: Improvement of bacterial blight resistance of "Minghui 63", an elite restorer line of hybrid rice, by molecular marker- assisted selection. Crop Sci 2000, 40:239-244.

33. Lian XM, Xing YZ, Yan H, Xu CG, Li XH, Zhang QF: QTLs for low nitrogen tolerance at seedling stage identified using a recombinant inbred line population derived from an elite rice hybrid. Theor App/ Genet 2005, 112:85-96.

34. Xue WY, Xing $Y Z$, Weng $X Y$, Zhao $Y$, Tang WJ, Wang L, Zhou HJ, Yu SB, Xu CG, Li XH, Zhang QF: Natural variation in Ghd7 is an important regulator of heading date and yield potential in rice. Nat Genet 2008, 40:761-767.

35. Fan CC, Yu XQ, Xing YZ, Xu CG, Luo LJ, Zhang QF: The main effects, epistatic effects and environmental interactions of QTLs on the cooking and eating quality of rice in a doubled-haploid line population. Theor Appl Genet 2005, 110:1445-1452.

36. Jiang GH, He YQ, Xu CG, Li XH, Zhang QF: The genetic basis of stay-green in rice analyzed in a population of doubled haploid lines from an indica by japonica cross. Theor Appl Genet 2004, 108:688-698.

37. Jiang $G H$, Hong $X Y, X u C G, L i X H$, He YQ: Identification of quantitative trait loci for grain appearance quality using a double-haploid rice population. J Integr Plant Biol 2005, 47:1391-1403.

38. Jiang DG, Lu S, Zhou H, Wu XJ, Zhuang CX, Liu YG, Mei MT: Mapping of the rice (Oryza sativa L.) thermo-sensitive genic male sterile gene tms 5 with EST and SSR markers. Chinese Sci Bull 2006, 51:417-420.

39. Li LZ, Lu KY, Chen ZM, Mu TM, Hu ZL, Li XQ: Dominance, overdominance and epistasis condition the heterosis in two heterotic rice hybrids. Genetics 2008, 180:1725-1742.

40. Temnykh S, DeCklerck G, Lukashova A, Lipovich L, Cartinhiur S, McCouch S: Computational and experimental analysis of microsatellites in rice (Oryza sativa $\mathrm{L}$.): frequency, length variation, transposon associations, and genetic marker potential. Genome Res 2001, 11:1441-1452.

41. Temnykh S, Park WD, Ayres NM, Cartinhour S, Hauck N, Lipovich L, Cho YG, Ishii T, McCouch SR: Mapping and genome organization of microsatellite sequences in rice (Oryza sativa L.). Theor Appl Genet 2000, 100:697-712.

42. McCouch SR, Teytelman L, Xu YB, Lobos KB, Clare K, Walton M, Fu BY, Maghirang R, Li ZK, Xing YZ, Zhang QF, Kono I, Yano M, Fjellstrom R, DeClerck G, Schneider D, Cartinhour S, Ware D, Stein L: Development and mapping of 2240 new SSR markers for rice (Oryza sativa L.). DNA Res 2002, 9:199-207.

43. Lincoln S, Daly M, Lander E: Constructing genetics maps with MAPMAKER/ EXP 3.0. Cambridge, Massachusetts, USA: Whitehead Institute Technical Report, Whitehead Institute; 1992

44. Wang DL, Zhu J, Li ZK, Paterson AH: Mapping QTLs with epistatic effects and QTL $\times$ environment interactions by mixed linear model approaches. Theor Appl Genet 1999, 99:1255-1264.

45. Boer MP, Wright D, Feng L, Podlich DW, Luo L, Cooper M, Fred A, Eeuwijk V: A Mixed-model quantitative trait loci (QTL) analysis for multiple environment trial data using environmental covariables for QTL-by- environment interactions, with an example in maize. Genetics 2007, 177:1801-1813.

46. Li ZK, Luo LJ, Mei HW, Wang DL, Shu QY, Tabien R, Zhong DB, Ying CS, Stansel JW, Khush GS, Paterson AH: Overdominant epistatic loci are the primary genetic basis of inbreeding depression and heterosis in rice. I. Biomass and grain yield. Genetics 2001, 158:1737-1753.

47. Van Ooijen JW: JoinMap ${ }^{\oplus}$ 4, Software for the calculation of genetic linkage maps in experimental populations. Kyazma BV, Wageningen, The Netherlands; 2006.

48. Rong JK, Feltus FA, Waghmare VN, Pierce GJ, Chee PW, Draye X, Saranga Y, Wright RJ, Wilkins TA, May OL, Smith CW, Gannaway JR, Wendel JF, Paterson $\mathrm{AH}$ : Meta-analysis of polyploid cotton QTL shows unequal contributions of subgenomes to a complex network of genes and gene clusters implicated in lint fiber development. Genetics 2007, 176:2577-2588.

49. Lu C, Shen L, Tan Z, Xu Y, He P, Chen Y, Zhu L: Comparative mapping of QTLs for agronomic traits of rice across environments by using a doubled haploid population. Theor App/ Genet 1996, 93:1211-1217.

50. Li J, Xiao J, Grandillo S, Jiang L, Wan Y, Deng Q, Yuan L, McCouch SR: QTL detection for rice grain quality traits using an interspecific backcross population derived from cultivated Asian (O. sativa L.) and African (O. glaberrima S.) rice. Genome 2004, 47:697-704.

51. Zhuang JY, Lin HX, Lu J, Qian HR, Hittamani S, Huang N, Zheng KL: Analysis of QTL $\times$ environment interaction for yield components and plant height in rice. Theor Appl Genet 1997, 95:799-808.
52. Zhou LJ, Chen LM, Jiang L, Zhang WW, Liu LL, Liu X, Zhao ZG, Liu SJ, Zhang LJ, Wang JK, Wan JM: Fine mapping of the grain chalkiness QTL qPGWC-7 in rice (Oryza sativa L.). Theor App/ Genet 2009, 118:581-590.

53. Xing YZ, Zhang QF: Genetic and molecular bases of rice yield. Annu Rev Plant Physiol Plant Mol Biol 2010, 61:421-442.

54. Lin WH, Xiao LT, Huang JL, Hong YH, Li HS: Relation between kernel chalkiness formation and source-sink characteristics in early indica rice. Acta Agr Nucl Sin 2003, 17:462-465.

55. Wan XY, Wan JM, Jiang L, Wang JK, Zhai HQ, Weng JF, Wang HL, Lei CL, Wang JL, Zhang X, Cheng ZJ, Guo XP: QTL analysis for rice grain length and fine mapping of an identified QTL with stable and major effects. Theor Appl Genet 2006, 112:1258-1270.

56. Asatsuma S, Sawada C, Kitajima A, Asakura T, Mitsui T: Alpha-amylase affects starch accumulation in rice grains. J App/ Glyco Sci 2006, 53:187-192.

57. Kang HG, Park S, Matsuoka M, Gynheung A: White-core endosperm floury endosperm-4 in rice is generated by knockout mutations in the C4-type pyruvate orthophosphate dikinase gene (OsPPDKB). Plant J 2005, 42:901-911.

doi:10.1186/1471-2156-15-49

Cite this article as: Peng et al:: Comparative mapping of chalkiness components in rice using five populations across two environments. BMC Genetics 2014 15:49.

\section{Submit your next manuscript to BioMed Central and take full advantage of:}

- Convenient online submission

- Thorough peer review

- No space constraints or color figure charges

- Immediate publication on acceptance

- Inclusion in PubMed, CAS, Scopus and Google Scholar

- Research which is freely available for redistribution 